\title{
Spinal cord injury management in Salisbury: the history of the Duke of Cornwall Spinal Treatment Centre
}

\author{
David Grundy ${ }^{1}$ and Jack Gardner ${ }^{2}$ \\ ${ }^{1}$ Consultant in Spinal Injuries; ${ }^{2}$ Executive Director, The INSPIRE Foundation, Duke of Cornwall Spinal Treatment \\ Centre, Salisbury District Hospital, Salisbury SP2 8BJ, UK
}

\section{Introduction}

It was only when Dr Ludwig Guttman was given the task of setting up a Spinal Injuries Unit at the then Ministry of Pensions Hospital at Stoke Mandeville in 1943 that a true revolution in the management of spinal cord injuries began in the United Kingdom. Thereafter, ten other spinal units with what we would now term 'a multidisciplinary approach' were gradually established round the country. However, the geographic spread was uneven with five of the six English units being situated in the Midlands and the North so that by the mid 1970s only two units were located in the southern part of the United Kingdom-Stoke Mandeville and Cardiff.

As might be expected as a result of the disparity, many patients faced long delays before being admitted to one or other of the then existing spinal units. Worse still, some spinal cord injured patients were not even given the benefit of the expertise afforded those who were fortunate enough to gain admittance to a spinal unit.

\section{Creation of the Spinal Injuries Unit at Salisbury}

In the late 1970s the then titled Department of Health and Social Security for the United Kingdom agreed that the service for spinal cord injured patients in the south of England was indeed inadequate, and, therefore, proposed the establishment of two new units, one to cover the needs of the South East and the other to service the South West. The Unit for the former area was placed at the Royal National Orthopaedic Hospital at Stanmore, a centre with a long-established orthopaedic tradition. In the South West it was acknowledged that the Regional Rehabilitation and Plastic Surgery Units at Odstock Hospital, Salisbury already had established strong reputations which greatly assisted in a successful bid to house the new Spinal Unit on the Odstock site.

Before deciding on the Unit-to-be's configuration, key members of the initial staff including the first Director, John Russell and the Nursing Administrator, Janet Egerton, visited Spinal Units in the USA,

Correspondence: D Grundy
Europe and Australia to provide a baseline design concept for the Salisbury Unit. The facility was ready for occupation in January 1984 and, 6 months later, His Royal Highness Prince Charles, Prince of Wales, Duke of Cornwall, accompanied by the then Her Royal Highness, Princess of Wales, opened the Unit declaring it to be The Duke of Cornwall Spinal Treatment Centre.

\section{Description of the Unit}

The Centre is housed in its own building originally designed as a 48-bed unit incorporating all the necessary support facilities and personnel needed to provide care for both outpatients and inpatients. Apart from a large downstairs activity/sports room (Figure 1) and a spacious swimming pool, the Centre is on one floor and entered by a lobby which serves as a reception area with offices for administrative and professional staff nearby.

The two wards have an eight-bedded area separated by a number of single patient rooms and flanked on their other extremities by three fourbedded patient rooms. The architect designed the interior so that all the patients occupy beds on the outer edge of the unit giving several patient areas with beautiful views to the surrounding hills. All the support facilities eg bathrooms, stores, sluice rooms, are on an inner triangle convenient to all the patient rooms. The patients have access to several inner 'courtyards' which are protected from the inevitable Wiltshire Winds! There is also a large, comfortably furnished, common room where patients, friends and relatives can talk, eat or relax at whatever time they choose. Of particular benefit is the availability of an Activities of Daily Living (ADL) flat which is used as a patient resource prior to moving from the hospital environment to the potentially traumatic first weekend at home following injury-an invaluable and necessary step prior to discharge.

Equally important are the occupational therapy and physiotherapy departments which are used by the patients who have progressed beyond the initial period of bed rest following their accident. The former facility is able to provide computer training to patients while 


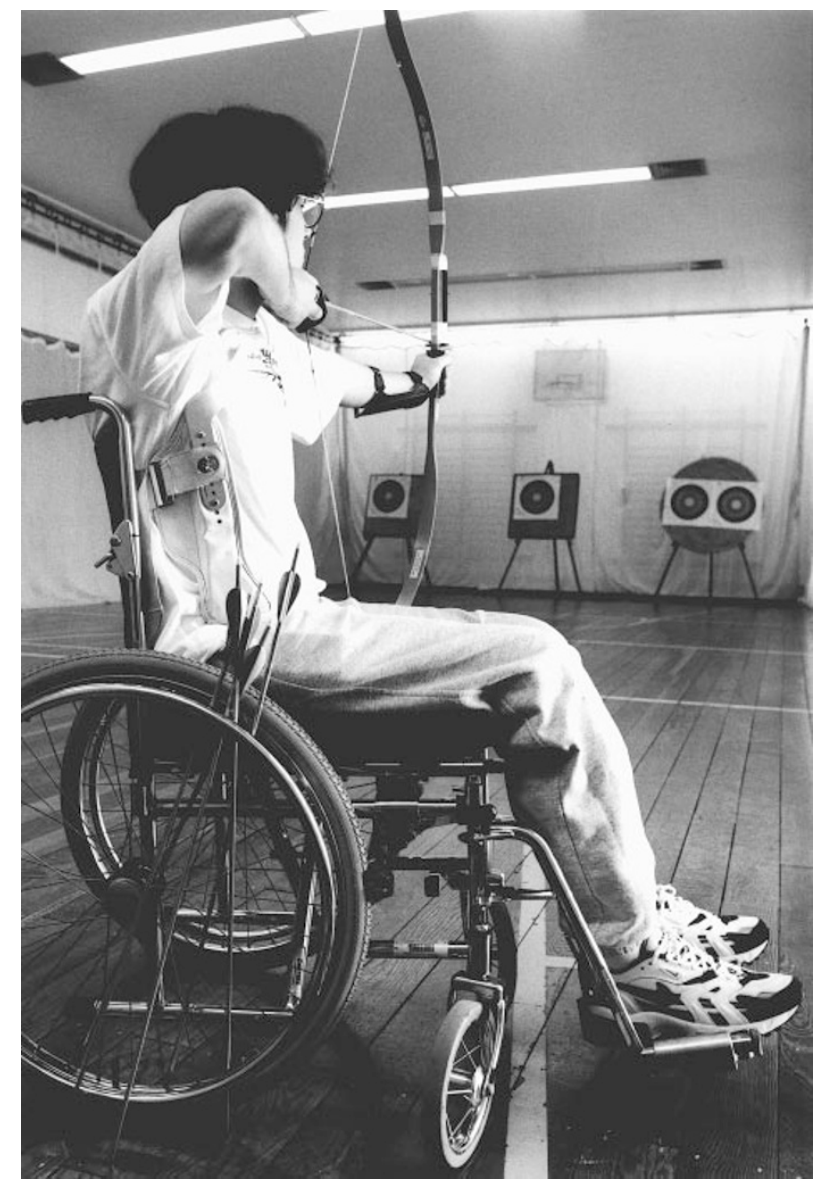

Figure 1 Sport and recreation also play an important part in the rehabilitation programme

the latter has the modern physiotherapy equipment needed to rehabilitate the patients.

Ambulance access is available on both upper and lower levels and the Unit has a helicopter landing site within 50 metres of the main entrance to the Unit (Figure 2). The Unit is, in the main, self contained and it is only when transferring patients to the operating theatres becomes necessary that leaving the boundaries of the Unit occurs.

\section{Philosophy of the Centre}

Back in 1984 the personnel who constituted the initial staff recognised that they were in a unique situation -a group of motivated and highly qualified professionals most of whom had not known each other before coming to Salisbury but had been given the task of creating 'from scratch' and with no tradition on which to fall back, a comprehensive Spinal Cord Injury Service for the Wessex and South West Regions, an area which housed over 6 million people.

From the earliest days a multidisciplinary approach has been the key to the functioning of the Unit. Furthermore it was soon recognised that newly injured

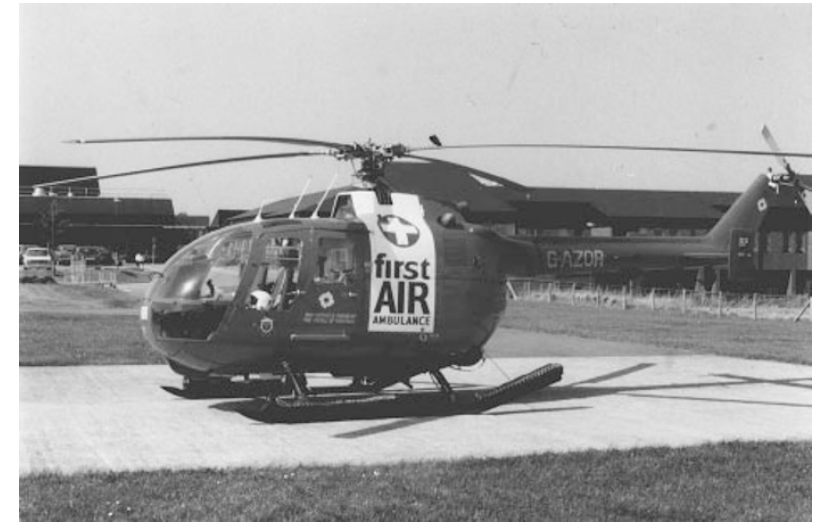

Figure 2 Helicopter on the Helipad with Spinal Unit in the background

patients would inevitably, and quite rightly, look on the Centre as their temporary home-from-home, designed to provide a relaxed, welcoming, and yet safe, atmosphere. Addressing this need would be, and still is, paramount to the Unit's philosophy of care. The patients would be given 'space' to express themselves and to come to grips with what would inevitably be a very emotional and stressful time.

\section{Staffing the Unit}

In June 1997, a six-bed self-contained unit with support rooms was built for patients who were virtually independent and nearing the end of their inpatient treatment. This expansion freed up beds in the main part of the Unit, so that we have been able to expand our service. Despite the increase to 54 beds, the official staffing level has changed little over the years in spite of the fact that the job has become harder, mainly as a result of the increased number of more seriously injured patients admitted to the Unit at an earlier stage after their injury.

Currently the staff consists of two consultants, three Senior House Officers, seventy nursing and nursing auxiliary grades, eight physiotherapists, five occupational therapists, two social workers, a ward administrator, two ward clerks, three community liaison coordinators (two nurses and a therapist), two radiographers and six secretaries.

\section{Patient mix}

The mix of patients has changed markedly in recent years in that the Unit deals with increasingly complex problems including ventilator dependent tetraplegics who require more resources than the paraplegic patients. Moreover, as more procedures and devices are designed to help non-critical spinal patients, readmissions continue to grow as does the demand for more complex solutions to the patients' medical and mobility problems. A typical in-patient population 
consists of 26 tetraplegics of which three are on long term ventilators and 28 paraplegics. Of this mix five are likely to be re-admissions for neurological procedures or for implant operations eg insertion of intrathecal baclofen pumps, sacral anterior root-stimulators, Cleveland 'Freehand' Systems. The average length of stay for tetraplegics is 7-10 months and for paraplegics $4-6$ months.

\section{Daily routine}

Patients are allocated a specific 'Primary Care Team' consisting of one person from each discipline who, within reason, stays with the patient throughout the time that he or she is on the Unit. Newly injured patients are given a bed in one of the two eight-bedded areas and, as they progress, are moved to one or other of the four-bedded side rooms. Patients requiring intensive care facilities are initially admitted to the Intensive Treatment Unit (ITU) in the adjacent General Hospital, undergoing shared care by the ITU and Spinal Unit staff. Strategic planning is agreed at the monthly Heads of Department Meeting which is chaired by the Unit Manager, currently the Senior Physiotherapist.

\section{Staff education}

In 1985, Salisbury became the second spinal unit to offer the English National Board ENB 371 course, 'Nursing Patients with Spinal Cord Lesions'. The Unit has a Lecturer/Practitioner shared with Bournemouth University. In addition to the ENB 371 6-month course, the Unit holds over 30 study days a year, mainly for nurses (including district nurses) carers, therapists and social services staff members. This is very much in keeping with one of the original aims of the Unit - to act as an educator in what, back in 1984 when the Unit opened, was, in many ways, virgin territory.

\section{Cooperation with other departments}

While the Spinal Unit is housed in its own building it does form an integral part of the General Hospital, now known as Salisbury District Hospital, which has all of the facilities of a large district general hospital, the only specialist services lacking being cardiothoracic surgery and neurosurgery services which are available 23 miles away in Southampton. The Unit is also most fortunate in having easy access to six other specialities essential to the overall team concept so necessary in comprehensively treating spinal cord injured patients:

Department of Orthopaedics: $\mathrm{Mr}$ John Carvell provides an excellent spinal surgery service.

Plastic Surgery Department: Mr John Hobby specialises in hand surgery for tetraplegics.

Theatres and Intensive Treatment Unit: The Spinal Unit relies on the services of the specialist personnel employed as part of the Main Theatres team with its recovery area. Adjacent to it is an Intensive Treatment
Unit, the staff of which are fully conversant with acute spinal cord injury care, in conjunction with the Spinal Unit Staff.

Department of Clinical Psychology: Dr Nigel North works with patients and their relatives to help resolve the difficulties experienced following spinal cord injury.

Department of Neurophysiology: Dr Jonathan Cole provides expertise in peripheral nerve conduction, EMG and magnetic brain stimulation.

Department of Medical Physics: The team headed by Dr Ian Swain provides expertise in Functional Electrical Stimulation (FES), involving both surface and implanted systems (see under 'Ongoing Research').

\section{Relationship with the Inspire Foundation}

The spinal research charity, INSPIRE, is located in the Spinal Unit itself. The charity provides funding for a range of spinal cord injury related research programmes and plans to fund the construction of a new facility (see under 'Future Plans').

\section{Cooperation with other spinal units}

The Spinal Unit maintains a cooperative alliance with the other units throughout the country in a variety of ways. Since its inception in 1984 the Unit has hosted the annual Guttmann Multi-Disciplinary Meeting on three occasions. The Unit liaises closely with personnel from Stanmore with respect to various research programmes. In conjunction with the Department of Medical Physics, the hospital hosted last year's National FES Conference of the Institution of Physics and Engineering in Medicine and Biology (IPEM). From the patients' perspective and the accompanying staff, this Unit continues to be a keen, and usually very successful, participant in the annual Stoke Mandeville Games. The various staff disciplines have regular meetings with their counterparts in other units.

\section{Cooperation with statutory and voluntary community services}

The Spinal Unit places great emphasis on the need to liaise and work with the patient and family, as well as statutory and voluntary organizations, to enable the patient to return to the community, and there successfully continue rehabilitation and adjustment. The Community Liaison Co-ordinators establish contact with local nursing services soon after admission. Likewise the social workers, who are employed by Wiltshire Social Services Department, refer the patient to his or her home social services department. The Spinal Unit occupational therapist and social worker then jointly visit the patient's home with staff from the local social services department to plan for home leaves, home adaptations and care needs on discharge. If rehousing is necessary, the social worker liaises with 
the relevant housing department in conjunction with the occupational therapist.

The Community Liaison Co-ordinators contact the community nursing service concerning the patient's nursing and care needs. Nursing, social services and care staff are invited to the Unit to be involved in planning for discharge and to learn care needs. The consultants inform the General Practitioner of the patient's home area, and a community case conference involving the patient, family, spinal unit staff, health and social services staff in the home area is arranged. Care management is usually undertaken by the home area.

The social workers undertake welfare benefits advice, negotiating with the Department of Social Security. They frequently put the patient in touch with the Spinal Injuries Association and other voluntary organizations which may be able to assist, to ensure that the patient and family are aware of resources and have as much knowledge, information and practical assistance as possible.

The Community Liaison Co-ordinators will normally visit the patient shortly after discharge, and continue to do so as necessary. They are also available to give advice and assistance to community staff and act as an expert reference point. Where appropriate they will refer back to any member of the multidisciplinary team. Patients also attend for regular outpatient review. They are seen by the consultant and pressure clinic personnel, and advice from all disciplines is available.

\section{Ongoing research}

\section{Functional electrical stimulation}

Three areas of research and associated developments have always been central to the work for spinal cord injured patients. For the past 2 years the Unit, in cooperation with the Charity INSPIRE and the Departments of Plastic Surgery and Medical Physics have offered to tetraplegics the practical possibility of having a useful restoration of hand control using the Cleveland 'Freehand' System. To date nine patients have benefited from such operations, performed by $\mathrm{Mr}$ John Hobby, the first of which was in April 1995 (Figure 3). The equipment for these operations is purchased and donated by INSPIRE from its research funds. It is noteworthy that five of the nine are patients from other UK spinal units.

In parallel with the upper limb implant programme, $\mathrm{Mr}$ Tony Tromans, consultant at the Unit has, in cooperation with a team from the Royal National Orthopaedic Hospital, University College London and the Royal London Hospital performed two successful lumbo-sacral anterior root stimulator implant (LARSI) operations at Salisbury. This procedure has allowed the first recipient to stand up from her wheelchair and to take as many as 23 consecutive, albeit tentative, steps using a walking frame (Figure 4).
The Unit also refers patients with incomplete spinal cord injuries to the Medical Physics Department for fitting of the 'Odstock' drop-foot stimulator which has already found a unique place in the treatment of

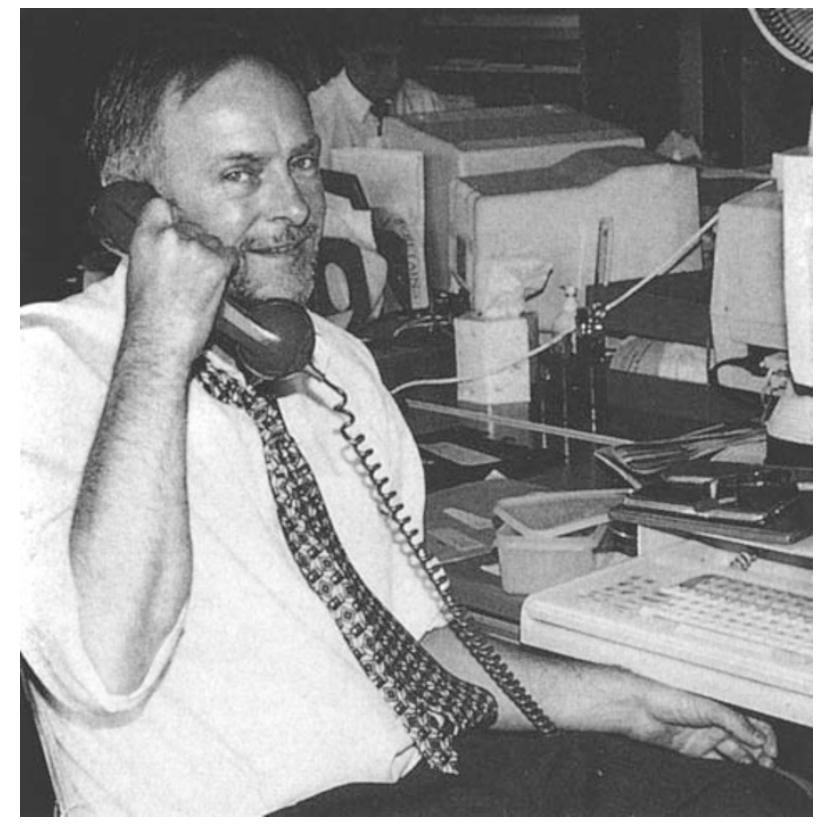

Figure 3 The first patient in Europe to have the Cleveland 'Freehand' system upper limb implant

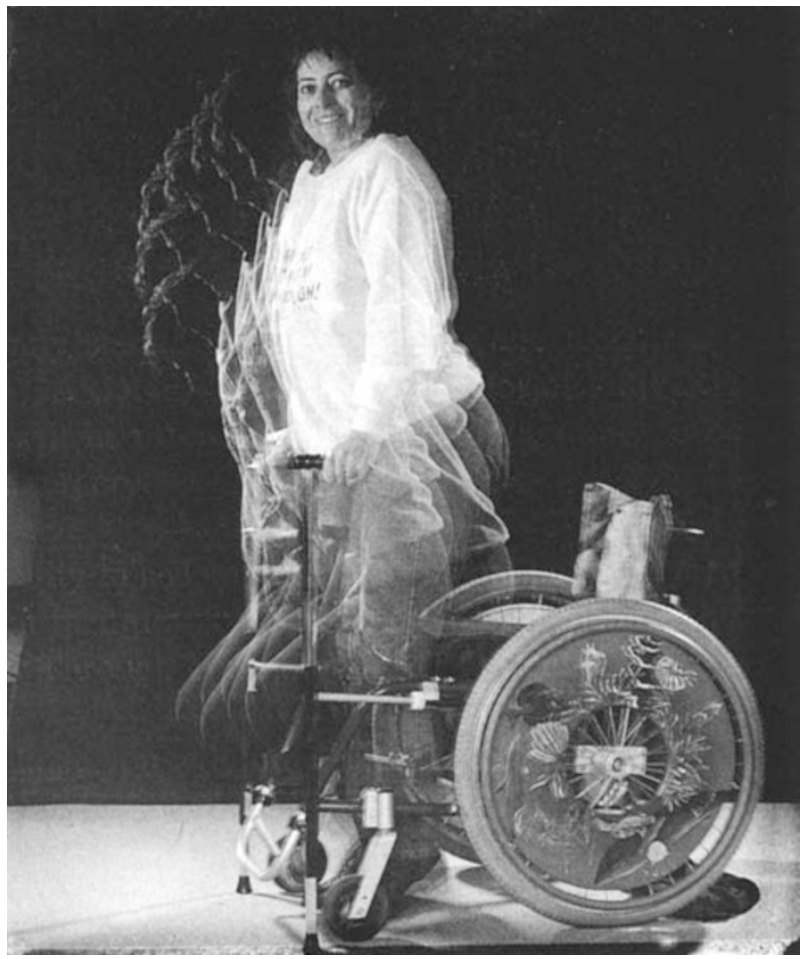

Figure 4 The first patient to receive a lumbo-sacral anterior root stimulator implant (LARSI) 
stroke patients, but which is also benefiting several of our spinal patients.

\section{Future plans}

Complementary facilities

As well as providing funds for the various operations INSPIRE has just launched an appeal to raise $£ 1500000$ to build a 'Mobility Through Technology' (MTT) Centre adjacent to the main spinal unit. The plan is to bring under one roof the research programmes, a conference and training centre, staff rooms and patient accommodation. The training centre will enable INSPIRE to have guaranteed spaces for the courses being held in Salisbury to train medical teams from around Europe in the operating techniques and the patient care aspects of the 'Freehand' implant system.

\section{Future research activities}

Continuation of both the Lumbo-Sacral Implant and Upper Limb Implant Programmes will be important.
Alongside these two key efforts will be other programmes, including the investigation of pain control using computerized sound and light programmes, further research into magnetic brain stimulation and work on advanced seating systems for the prevention of pressure sores.

\section{Summary}

One point remains paramount in our minds and that is, in order to achieve the goals we have set, we need vigorously to ensure that Spinal Units continue to be seen as the only facilities competent to provide for the diverse needs of spinal cord injured patients. We at the Duke of Cornwall Spinal Treatment Centre are fortunate in having a first class, expanding facility and have the tireless cooperation of both our staff and those of the other departments in Salisbury. We recognize also that we can play a leading role in the treatment of patients, not only from the Southwest, but, where and when appropriate, from further afield. 